\title{
Potential Applications of functional Magnetic Resonance Imaging (fMRI) to Organizational Research: A Primer and Sample Study
}

\author{
Allen I. Huffcutt \\ Bradley University \\ Wen-Ching Liu \\ Saint Francis Medical Center \\ Lori A. Russell-Chapin \\ Bradley University
}

Follow this and additional works at: https://scholarworks.bgsu.edu/pad

Part of the Human Resources Management Commons, Industrial and Organizational Psychology

Commons, and the Neuroscience and Neurobiology Commons

How does access to this work benefit you? Let us know!

\section{Recommended Citation}

Huffcutt, Allen I.; Liu, Wen-Ching; and Russell-Chapin, Lori A. (2018) "Potential Applications of functional Magnetic Resonance Imaging (fMRI) to Organizational Research: A Primer and Sample Study," Personnel Assessment and Decisions: Number 4 : Iss. 2 , Article 4.

DOI: https://doi.org/10.25035/pad.2018.02.004

Available at: https://scholarworks.bgsu.edu/pad/vol4/iss2/4

This Measurement and Measures is brought to you for free and open access by the Journals at ScholarWorks@BGSU. It has been accepted for inclusion in Personnel Assessment and Decisions by an authorized editor of ScholarWorks@BGSU. 


\title{
Potential Applications of Functional Magnetic Resonance Imaging (fMRI) to OrganizationaL Research: A Primer and Sample Study
}

\author{
Allen I. Huffcutt ${ }^{1}$, Wen-Ching Liư ${ }^{2}$, and Lori A. Russell-Chapin ${ }^{1}$ \\ 1. Bradley University \\ 2. Saint Francis Medical Center
}

ABSTRACT

The first purpose of this manuscript is to provide a primer for organizational researchers on both fMRI and brain physiology because few are likely to have encountered an in-depth treatment of either previously. The second purpose is to present the results of an actual fMRI study on an organizational topic (structured employment interviews) as a sample to help illustrate the potential of this type of research. Results of the sample study enhanced understanding of the brain processes behind responding to situational (SI) and behavior description (BDI) interviews, and offered several promising directions for follow-up research. To illustrate the latter, there appears to be separate region of the brain for handling complex social situations, which was activated only in the SI scans. This region could help explain the common use of the impression management tactic ingratiation in Sls. Given the emerging trend for larger universities to acquire fMRI equipment for research purposes, this type of research may be more viable then ever for organizational researchers.

fMRI, job applicant interviews, situational interviews, behavior description interviews
As highlighted by a special commemorative issue of the Journal of Applied Psychology in March of 2017, organizational research, particularly selection and assessment, has now reached its century mark. A variety of conventional research methodologies have been employed over these decades to test a myriad of organizational hypotheses. This research was largely a combination of traditional correlational analysis and designs based on group difference (e.g., ANOVA), supplemented by the advent of meta-analysis (Hunter, Schmidt, \& Jackson, 1982).

Although conventional research methodology has provided considerable insight into organizational issues and dynamics, it does have its limits. These methodologies typically provide information on surface features and relationships, such as the association between general mental ability and job performance (e.g., Schmidt \& Hunter, 1998). Even studies that have attempted to investigate "inner" processes such as decision-making tendencies of interviewers (e.g., the Brunswick Lens Model; Gifford, Ng, \& Wilkinson, 1985) still relied on outer manifestations such as interviewer ratings to make their inferences. It would seem that new approaches that provide more direct insights into the underlying mental processes that initiated and led to surface outcomes could prove valuable and could accelerate research progress greatly.
Physiological-based approaches (some of which are relatively new) have significantly enhanced understanding in a number of other areas in the behavioral and medical sciences. For instance, clinical researchers commonly find that self-report measures of the personality trait neuroticism have limited success in predicting actual behavioral outcomes such as difficulties with mood, vigilance, and attention (Matthews \& Gilliland, 1999). Eisenberger, Lieberman, and Satpute (2005) measured neurological activity in the brain associated with neuroticism directly and found that it predicted outcomes better than self-report. Similarly, Russell-Chapin, Kemmerly, Liu, Zagardo, and Chapin (2013) found identifiable changes in the fMRI brain scans of ADHD children provided with neurofeedback (relative to a control group of ADHD children), ones that corresponded with more normal functioning.

Recognition of the potential importance of physiological-based perspectives has begun to emerge within industrial and organizational psychology. In July of 2013, for instance, M. K. Ward and B. Becker began a column on organizational neuroscience $(\mathrm{ON})$ in The Industrial-Or-

Corresponding author:

Allen I. Huffcutt

Email: huffcutt@fsmail.bradley.edu

Phone: 309-251-9623 
ganizational Psychologist (TIP). Since then, topics such as leadership development (Ward \& Becker, 2014), counterproductive work behaviors (Ward \& Becker, 2015), and entrepreneurship (Ward, 2017) have been viewed from a neuroscience perspective (see Zhu, Ward, \& Becker, 2016, for a summary).

Two physiological approaches in particular appear to have the most potential to advance organizational research. One is electroencephalographing (EEG), which involves placing small electrodes on the scalp that detect and chart neural activity. Although EEG picks up on brain activity almost instantaneously, its level of precision is limited and it only captures activity on the very outer surface of the brain (Freeman, 2004). The traditional output of EEG is a chart with a line showing the amplitude and frequency of the brain waves involved. The other, which is newer and overall more promising, is functional magnetic resonance imaging or fMRI. Although the equipment is considerably more expensive, fMRI is much more precise than EEG and can provide rich and detailed 2D and 3D images of neural activity across all parts of the brain.

Despite its potential, to our knowledge fMRI has not been used to assess organizational phenomenon directly. Rather than transferring and generalizing fMRI findings from other areas, it would seem advantageous for organizational researchers to begin to do their own investigations. A number of larger universities now possess fMRI equipment dedicated solely for research, making access to this equipment easier than ever. Direct use of fMRI could not only help advance existing lines of research but also open up entirely new ones.

This manuscript has three purposes. The first is to provide an overview (primer) of the fMRI technique. Few organizational researchers have had exposure to fMRI, and a brief overview of how it works could prove helpful. The second is similar, which is to provide a primer on brain physiology, with a special emphasis on applicability to organizations. The third and final purpose is to present results from an actual fMRI study as an example of the potential of this type of work, one conducted to assess and compare the two main types of modern structured interviews. This study should help illustrate how fMRI can be used to assess important organizational phenomenon.

\section{Primer on fMRI}

\section{Magnetic Resonance Imaging (MRI)}

In the medical area, MRI formally emerged in the early 1970 s as an alternate to radiation-based diagnostic techniques. Paul C. Lauterbur is created with its invention, although others had proposed underlying theories and even preliminary equipment designs previously (see Dawson, 2013; Rinck, 2001). Lauterbur was given the credit mainly because he was the first to develop a way to create $2 \mathrm{D}$ and
3D images. He, along with another individual working in this area (Sir Peter Mansfield), were awarded the Nobel Prize in Physiology or Medicine in 2003.

Typically, the same equipment is used in functional magnetic resonance imaging (fMRI), which is why behavioral science research using fMRI is frequently conducted at hospitals. The primary difference is that participants are given some type of task or activity (i.e., function) to do while being scanned. For instance, to assess spatial activation, participants could be shown figures on a screen and asked to press a button when a square is presented. Compared to medical MRI, fMRI tends to be done with lower spatial resolution but greater scanning frequency (Thulborn, Waterton, Matthews, \& Radda, 1982), but again, it is the same equipment.

The theory behind fMRI is grounded in utilization of brain resources such as sugars (e.g., glycogen) and oxygen (Baars \& Gage, 2010; Gailliot, 2008). When a certain part of the brain is active, its neurons consume these resources. Oxygen depletion is the resource that is the most relevant to fMRI. In response to its depletion, the vascular system ramps up to meet the increased demand, which, in fMRI terminology, is measured as blood-oxygen level dependent (BOLD) activity. There is roughly a 6-second delay between the occurrence of such activity and its appearance on fMRI scans.

As a point of reference, MRI equipment bears some similarity to computerized tomography (CT), a radiation-based technique that also emerged in the early 1970s. CT takes X-rays from a variety of different angles, which are then integrated to form detailed images of both hard and soft tissue. Sample MRI and CT machines are shown in Figure 1. Both have a doughnut-shaped configuration, although the cavity is noticeably deeper in MRI (which is why participants are often screened for claustrophobia). Both have a bed that participants lie upon that transports them inside the machine. MRI has a head restraint as well because its scanning is more sensitive to movement. MRI takes eonsiderably longer, up to 30 minutes (and sometimes more depending on the nature of the research), and is surprisingly loud (participants typically wear ear protection). In contrast, $\mathrm{CT}$ can take less than 5 minutes and is relatively quiet. Although CT could be used for behavioral science research, and would have several advantages in doing so (e.g., duration, noise level), the use of radiation generally makes it prohibitive.

As a point of medical interest, CT and MRI can generally be used interchangeably, and together form the basis for modern high-definition scanning. That said, CT tends to be better for viewing bone injuries, diagnosing lung and chest problems, and detecting cancer. Conversely, MRI is better suited for examining soft tissue in ligaments and tendons, spinal cord injuries, and brain tumors (see http:// www.diffen.com/difference/CT_Scan_vs_MRI). 
FIGURE 1.

A modern MRI machine is shown on the left, a modern CT on the right.
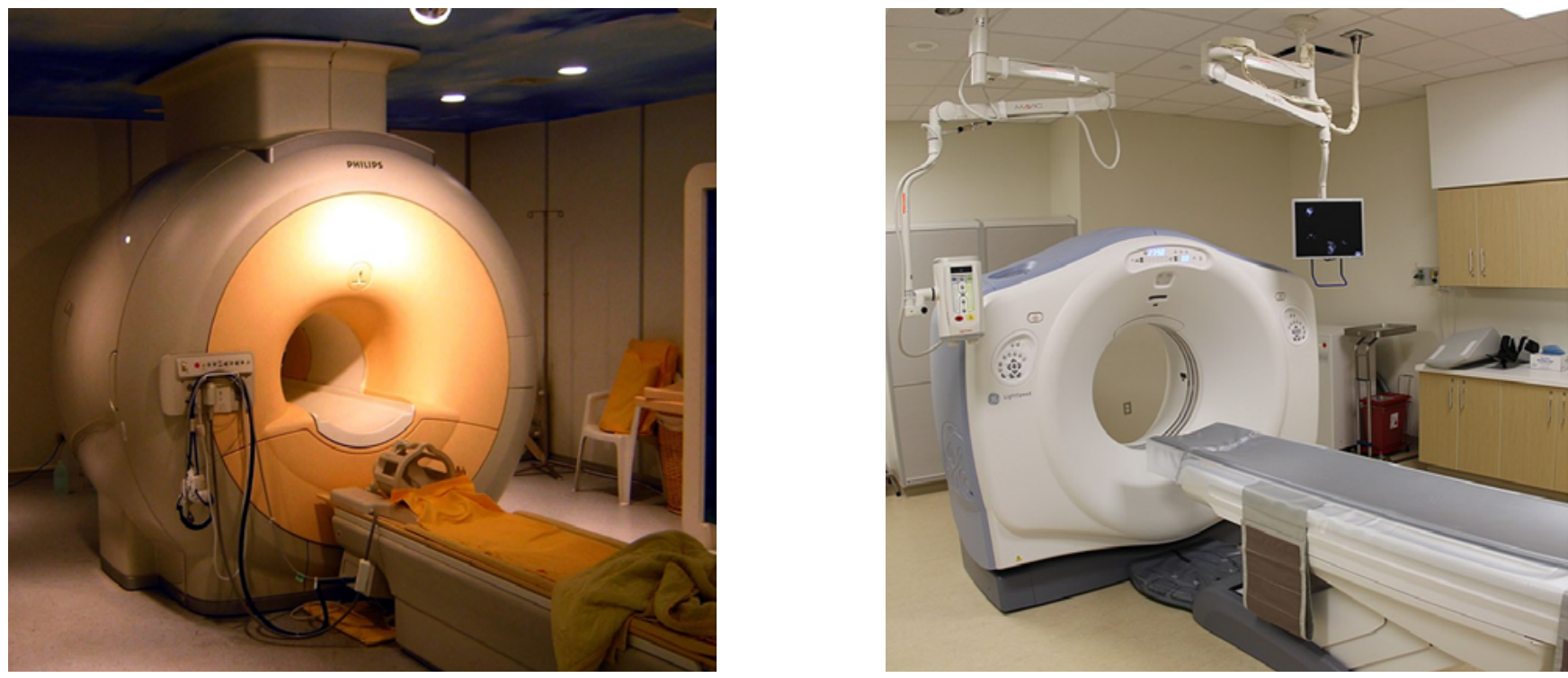

The MRI image was obtained from the website https://en.wikipedia.org/wiki/Magnetic_resonance_imaging. As noted in the links associated with this image, its creator, KasugaHuang, has granted permission to use it freely under the terms of the GNU Free Documentation License (see https://commons. wikimedia.org/wiki/File:Modern_3T_MRI.JPG). The CT image was obtained from https://en.wikipedia.org/wiki/CT_scan\#/media/File: UPMCEast_CTscan.jpg. Similarly, its creator, daveynin, has authorized free usage under the Creative Commons Attribution 2.0 Generic License (see https://commons. wikimedia.org/wiki/File:UPMCEast_CTscan.jpg). Both images were cropped slightly to enhance simultaneous presentation, which is permitted under their respective licensing platforms.

\section{Primer on Brain Physiology}

\section{Organization of the Brain}

Before proceeding to the fMRI study itself, we provide a general discussion of the structure and layout of the brain, which we feel is important given how new neuroscience is to the organizational area. A topographical map of the brain is provided in Figure 2. The top part of this figure is an outside view of the left hemisphere (i.e., side of the brain), whereas the bottom part shows a "sagittal" (front-to-back) and "medial" (middle) slice of that hemisphere. Superimposed on these figures are common subdivisions known as Brodmann areas (Brodmann, 1909), which are localized regions associated with specific mental functions. Currently, over 100 such areas have been identified (see Garey, 2006, for a detailed description of these areas).

Although not explicitly identified as such, Figure 2 also illustrates the four major lobes in the brain. The frontal lobe (FL) comprises Brodmann areas 4 and 6 and all areas forward from them. The parietal lobe (PL), one function of which is thought to be high-level sensory integration, includes areas 3,1 , and 2, and surrounding areas behind them. The occipital lobe (OL), the visual center, includes areas 17, 18, and 19. Finally, the temporal lobe (TL) is the "arm" that juts out to the side and includes areas 20, 21,
22, and 38. As discussed shortly, in addition to auditory processing, the inner structures of the TL play a key role in human memory.

Of particular relevance to this investigation is the further subdivision of the frontal lobe into two primary areas. The top, back-most portion is the motor cortex, an area associated with higher level motor functions and includes areas 4 and 6 . The front-most part is the prefrontal lobe (PFL), and it includes areas 8, 9, 10,11,12, 13, 44, 45, 46, and 47 (see Fuster, 1997). The PFL is the seat of the highest levels of human functioning, including key mental operations such as setting objectives, forming goals, devising plans of action, following social and moral standards, impulse control, and even personality (Miyake, Friedman, Emerson, Witzki, $\&$ Howerter, 2000).

Also of particular relevance to this investigation is the temporal lobe, which is traditionally understood to be involved in auditory processing. Lessor known is that it also contains an inside structure that is the primary center for human memory. Specifically, the hippocampus ${ }^{1}$ runs roughly through the middle of the TL and is surrounded by the

\footnotetext{
1 Of scientific interest, the term hippocampus is a derivative of two Greek words that combine to form seahorse. Although there are some noticeable differences, the hippocampus does bear a distinct resemblance in shape to this unique sea creature.
} 
FIGURE 2.

Map of the cortex from Baars and Gage (2010), Figure 12.3 (p. 403). Reproduced with permission of the publisher.
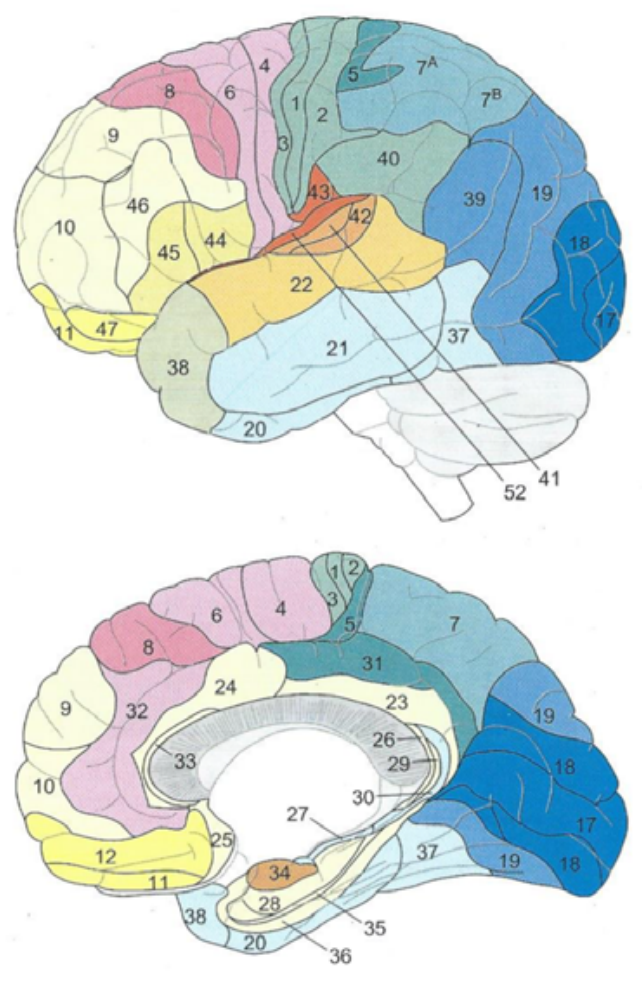

parahippocampus. This region appears to be the primary area for both transferring experiences into long-term memory and recalling those experiences at a later point (Diana, Yonelinas, \& Ranganath, 2007).

However, it is important to note that the hippocampal region does not store the actual memory traces (fragments, elements) but rather just processes them. Storage of longterm experiences, known as autobiographical or episodic memory in the cognitive literature, is thought to occur through alteration of synaptic connections (including formation of new ones) throughout the outer surface of the brain (Synapses are the junction point between neurons.). Basic visual elements, for instance, could be stored in the occipital lobe, auditory ones in the outer surface of the temporal lobe.

When an attempt is made to recall an experience, the hippocampal region guides the search for, and integration of, scattered memory fragments. There appears to be intermediate "processing stations" of sorts that help integrate base memory elements. For instance, the posterior (rearward) part of the parietal lobe (including Brodmann areas $5,7,39$, and 40) appears to be involved in recall and integration of contextual details, visual features of the people involved (Epstein \& Kanwisher, 1998), and auditory information such as sounds or someone speaking (cf. Cabeza, Ciaramelli, Olson, \& Moscovitch, 2008).

\section{Sample fMRI Study}

\section{Employment Interviews}

Research has shown that structuring employment interviews tends to improve both their reliability and validity relative to traditional, unstructured ones (see Huffcutt, Culbertson, \& Weyhrauch, 2013, 2014). In fact, highly structured interviews appear to provide a level of criterion-related validity that is on par with the best available predictors of job performance (e.g., cognitive ability, job knowledge; see Schmidt \& Hunter, 1998). Moreover, they tend to do so with minimal impact on protected groups (Huffcutt \& Roth, 1998).

Within the realm of structured interviews, two question types have become particularly popular and used commonly across organizations. One is the situational interview or SI (Latham, Saari, Pursell, \& Campion, 1980), in which candidates are presented with a series of hypothetical, job-related scenarios and asked to indicate how they would respond if hired and confronted with those situations. The other is the behavior description interview or BDI (Janz, 1982), where candidates are asked to describe real experiences from their past where they demonstrated attributes and capabilities important for the desired position. These two formats are attractive because they not only provide strong criterion-related validity (see Taylor \& Small, 2002) but are based on types of interview questions with which both employers and applicants appear to be comfortable.

However, a recent meta-analysis found a mean correlation of only .47 between SI and BDI questions that were carefully developed to assess the same job attributes and written from the same base of critical incidents (a within-study design), and this was after correction for measurement error in the interviews (Culbertson, Weyhrauch, \& Huffcutt, 2017). Their lack of strong correspondence is surprising because there appears to be an implicit assumption in both the literature and professional practice that the two types of questions are, for the most part, interchangeable.

Scattered empirical evidence tends to support the modest SI-BDI correlation and the possibility of unique construct measurement. For instance, a recent study found evidence for a BDI method effect, one rooted in the interaction between the personality traits ambition and sociability (Huffcutt, Culbertson, \& Goebl, 2015). Method effects have been found in other selection predictors that are method rather than construct based (e.g., assessment centers; see Kuncel \& Sackett, 2014). To date, no evidence for a method effect for SIs has been found. Further, job complexity appears to moderate the validity of SIs but not BDIs (Huffcutt, Conway, Roth, \& Klehe, 2004). 
The lack of construct understanding has significant implications for the practice of structured interviewing. Use of a test battery in selection is both popular and advantageous psychometrically, but it is unclear at the present time whether it is sufficient to use one question type or the other or whether both should be utilized, and if both, whether the two sets of ratings should be combined or kept separate. At a more general level, making continued improvements and refinements to the SI and BDI techniques is problematic without first having a reasonable understanding of how they function and what they tend to assess.

Theoretically, there is very little in the interview literature that could help explain the lack of strong correspondence between matching SI and BDI questions. SIs are built upon the premise of goal setting (Latham et al., 1980), specifically that the intentions stated by candidates are thought to reflect personal goals, which in turn are an immediate precursor to actions. Conversely, BDIs are built upon a behavioral consistency premise, specifically the notion that past behavior is the best predictor of future behavior (Janz, 1989). Both of these theoretical bases have merit, but they are general tenets and provide little information about underlying processes and mechanisms involved in SI and BDI responding and also about the factors that can exert influence upon them.

In summary, conventional research methodology appears to have taken us only so far in our understanding of these two unique approaches to employment interviewing. Continued research of this type is unlikely to provide major advances, at least not in the near future. Given the almost universal prevalence of employment interviews and the popularity of these two structured formats, it may indeed be time to consider alternate research methodologies.

As noted earlier, the primary purpose of this study is to utilize fMRI to enhance understanding of SI and BDI responding. To aid in this endeavor, we identified specific Brodmann areas that could be relevant to both types of questions. Those areas are presented in Table 1. As a point of qualification, areas that are likely to be involved in all interview formats are excluded from this table, including Wernicki's area (22) for speech comprehension. This area is should be involved in listening to the questions as they are read regardless of the type.

\section{Participants}

Ten individuals ( 5 men, 5 women) were recruited from the institution with which the first author is affiliated. They were all undergraduates of normal college age and were involved in research labs under various departmental faculty. In contrast to typical college samples motivated simply by extra credit, we believed they would put forth their very best effort. Our subjective observations were that this was in fact the case. All participants had at least some job experience in general retail sales (e.g., mall clothing stores), which is what the interview questions were developed to represent. Further, all were right handed, selected as such to avoid a confound from handedness influences. For instance, right-handed people tend to use the left side of their brain for primary functions (e.g., language), whereas those who are left-handed often utilize both sides (see Nisiyama \& Ribeiro-do-Valle, 2014).

In regard to sample size, 10 was the maximum number allotted to us by the fMRI coordinating organization of the host hospital. Although such a sample size may seem small in comparison to typical organizational research, it is not atypical with fMRI given its high cost and often limited availability of equipment and technical staff (particularly in hospitals). Desmond and Glover (2002), for example, noted

TABLE 1.

Brodmann areas of particular relevance to BDIs and SIs

\begin{tabular}{lll}
\hline Area & Name & Description \\
\hline & Most relevant to the behavior description interview \\
10 & Anterior prefrontal cortex (A-PFC) & \multicolumn{1}{l}{ Strategic processes in memory recall, working memory, multitasking } \\
$5,7,39,40$ & Parietal lobe & Higher level sensory integration \\
23,31 & Posterior cingulate & Episodic memory recall \\
& Most relevant to the situational interview & \\
9,46 & Dorsolateral prefrontal cortex (DL-PFC) & Working memory, mental flexibility, planning, inhibition, motor planning, deception \\
$11,12,47$ & Orbitofrontal cortex (OFC) & Emotion, reward in decision making, impulse control, sensory integration \\
$24,32,33$ & Anterior cingulate & Reward anticipation, decision making, impulse control \\
\hline Note. ${ }^{\text {a }}$ Part of the ventromedial prefrontal cortex (V-PFC); “orbit” is in reference to its position over the eye sockets.
\end{tabular}


that about 12 subjects were needed to achieve $80 \%$ power for typical activations. Our sample size was only slightly lower than this value. Further, as outlined later, our results were strong and seemed to converge very nicely across participants, lending strength and credibility to the findings.

\section{BDI and SI Questions}

An interview containing seven sets of matching BDI and SI questions (14 total) was developed to reflect general, entry-level retail employment. To illustrate, one SI question presented a scenario where a fellow employee comes into work very withdrawn and irritable, and later that day, blows up over something small. Participants were then asked what they would do if they were hired and faced with this situation. The matching BDI question prompted participants to think of a time when they had to deal with someone who had become withdrawn and irritable for no apparent reason, even to the point of blowing up over small things. They had to describe the situation, the actions they took, and the outcome.

\section{Procedure, Equipment, Software, and Protocol}

Participants were screened to ensure that they did not have metal implants anywhere in their body (because MRI machines are essentially giant magnets), neurological illness, previous or current head trauma, or claustrophobia. Female participants were tested to confirm they were not pregnant. They were then assigned a day and time for testing at the host hospital. Scanning was done using a General Electric Signa 3 Tesla whole body scanner, a device that was used for actual medical purposes for most the week. The acquired fMRI data were processed by the second author using the FSL 4.1 software program, which is part of the FMRIB Software Library (v5.0) created by the Analysis Group (Oxford, UK).

In terms of protocol, the seven BDI questions were presented first, followed by the seven SI questions. To provide a baseline (off) condition, there was a gap between questions where simple musical notes were played. The decision of order turned out to be a more difficult one than we had anticipated. Presenting the questions in blocks by type was advantageous because it helped reinforce and extend the mental processes associated with each interview format. However, presentation by intended dimension would have allowed matching sets of BDI and SI questions to be presented together. Given that the focus of this investigation was on brain processes (rather than assessment of specific job dimensions), we choose the block option. This decision to use the block option was made even more difficult by the limitation that we could not counterbalance and present the SI block first to half of the subjects. Doing so would have necessitated the creation of a second fMRI protocol, which was prohibited given that the study was done in a hospital and we had limitations on both time and staff resources.
Thus, the BDI questions were presented first simply out of convenience. Given the outside possibility that the actual experiences recalled via the BDI questions served as a primer for intended actions in the SI scenarios, we strongly encourage follow-up research where SI questions are presented first. We also encourage research that utilizes dimensional presentation.

Procedurally, participants were provided with each question in turn via a display. Given the level of noise associated with an fMRI machine, reading the questions orally was not feasible (The intervening musical notes were presented via headphones). We note that Wernicke's area, which is responsible for speech comprehension (Kennison, 2013), was highly activated with both sets of questions. The implication of this activation is that our participants were processing the questions in a very similar manner to having them read. Also because of the noise, participants were asked to form their responses mentally instead of stating them verbally. There was strong activation in Brocca's area, the region responsible for speech production (Kennison, 2013), suggesting that participants were forming verbal sentences as they would normally, just not speaking the actual words (Scans for both of these areas are available from the first author.).

\section{Psychometrics of fMRI Scanning}

Given how new fMRI is to organizational research, we felt it important to provide a brief summary of the psychometric process by which individual scans are synthesized into a single, collective one. Unlike psychological testing (e.g., mental, personality) where the assessment itself is standardized, there is an initial problem in scanning. Simply put, everyone's head (and brain) is of different size. Fortunately, the various structures and lobes are in the same relative position, thereby making it possible to standardize their locations. Thus, one of the first steps in fMRI is to standardize individual scans using three-dimensional MNI (Montreal Neurological Institute) coordinates (Poldrack, Mumford, \& Nichols, 2011; see also Talairach \& Tournoux, 1988).

Once standardized, the three-dimensional brain space is divided up into small cubes called "voxels." The size (dimensions) of the voxels is determined by the sophistication of the equipment and the settings selected. Four to five millimeters is common, but voxel size can be small as one millimeter (Huettel, Song, \& McCarthy, 2009). Each voxel contains a few million neurons and tens of billions of synaptic connections.

The strength of the BOLD signal is then assessed in each voxel. This is accomplished using conventional significance testing methodology. Essentially, a $t$ value is computed for each voxel by dividing the difference in signal strength between the "on" or experimental condition (here the interview questions) and the "off" or control condition 
(here musical notes) by the amount of noise present (expressed as a standard deviation (see http://blogs.discovermagazine.com/neuroskeptic/2010/08/19/fmri-analysis-in1000 -words/\#.WsuB5UxFxPa). An overall $t$ value for each voxel is then formed by averaging across participants and, by common fMRI convention, transformed to a $z$ value. True activation is assumed only if the resulting $z$ value reaches statistical significance (i.e., $p<.05$ ).

Graphically, 3D images are formed by cumulating significant voxel activation across the entire brain (e.g., see Figure 3). Intensity levels are displayed using colors. Red is at the low end and yellow is at the high end, and they reflect $z$ values of 2 and 5 respectively. Orange reflects intermediate levels of intensity (Readers may recall that the twotailed, .05 critical of the $z$ statistic is 1.96 . This rounds to 2 as shown in Figures 3-5.).

One last psychometric issue worthy of mention is individual differences. Similar to group-based experimental designs (e.g., ANOVA), the focus in fMRI is on average effects and not on within-group variability. Within-group variability plays an important role in ANOVA, for instance, as the higher the within variability the greater the mean separation needed for the $F$ value to reach significance. Likewise, wide individual differences in the degree of activation for a given voxel reduce the overall (average) strength of the BOLD signal and makes it harder to reach the established threshold of activation corresponding to $p<.05$. As shown in Figures 3-5, our scans included a number of activations shaded orange (and even yellow) with corresponding $z$ values commonly in the 3-4 range. Such magnitudes suggest a reasonably strong degree of consistency across participants.

\section{RESULTS $^{2}$}

\section{Common Activation Areas}

$D L-P F C$. Both type of questions showed strong activation in a prefrontal region known as the dorsolateral prefrontal cortex or DL-PFC, which is shown in Figure $3^{3}$ This area corresponds mainly to Brodmann Area 9, although there may have been some activation in Area 10 as well. As noted in Table 1, this area seems to be involved in management of high-level mental processes such as holding information in working memory, analyzing and manipulating that information, strategically arriving at decisions, and even consideration of deception. What does not appear to be one of its primary functions is interpersonal handling of complex social situations (Miller \& Cummings, 2007; p. $355)$, which seems to be different than processing of general information.

Hippocampal region. Given that the fundamental nature of BDIs is recall and description of past experiences relevant to the position (e.g., "Tell me about a time when you..."), hippocampal activation was expected. There in fact was strong BDI activation in both the hippocampus and parahippocampus. What is interesting with this finding is that it occurred on both the left and ride side, confirming the central role that memory recall plays in the BDI process. In contrast, a majority of the findings from this study involved activation only on the left side (which was expected given that the participants were right handed).

What was less certain upfront was the strength of the activation in the hippocampal region with the SI. Given its purely hypothetical nature, participants could, in theory, respond without ever tapping into long-term memory or doing so minimally. For instance, the DL-PFC by itself seems well-equipped to consider a SI question and strategically arrive at an intended action. Then again, memory search is such an integral part of human functioning that participants may still have had a tendency to do so even with the hypothetical questions.

Results did in fact indicate SI activation in the left parahippocampus, a region that is thought to play an important role in memory storage (encoding) and retrieval particularly with contextual scenes (i.e., physical surroundings rather than people or faces [Mégevand, et al., 2014]). Unlike the BDI, which appears to be more media rich (discussed in the next section), this finding could suggest that the memory processes participants engage in with the SI tend to be more basic. BDI and SI activation in the hippocampal region is shown in Figure 4.

\section{Unique Activation Areas}

Behavior description interview (BDI). The combination of multiple areas of activation across varied parts of the outer cortex suggests that recall of past experiences with the BDI is media rich. Within the occipital cortex, the visual processing center of the brain, there was activation in the fusiform gyrus (left and right side; Bodman area 37) and lingual gyrus (both left and right) areas. The former is involved with complex visual images (Machielsen, Rombouts, Barkhof, Scheltens, \& Witter, 2000), and the latter with color, face, and body recognition (Weiner \& Grill-Spector, 2010; Trafton, 2012). There was also activation in the frontal lobe regions that pertain to motor functions (e.g., planning, control, and execution), including the precentral gyrus (located in the frontal lobe), right cerebellum, substantia nigra, pallidum, and putamen (see DeLong et al., 1984). Collectively, these findings suggest that mental recall of past experiences may contain not just faces and details, but scenes, images, colors, and even motion. Scans showing these areas of activation are available upon request from the first author.

\footnotetext{
2 Given that our fMRI study is presented mainly as an example of the potential applicability of $\mathrm{fMRI}$ research to organizations, we only provide select results in this manuscript. Full results are avail able from the first author.

${ }^{3}$ Because of the manner of reporting with the software, the right side of the figure actually portrays the left side of the brain.
} 


\section{FIGURE 3.}

As indicated by the arrows, behavior description interview activation in the left dorsolateral prefrontal (DL-PFC) is shown in Panel (a), whereas situational interview activation in the left DLPFC is shown in Panel (b).

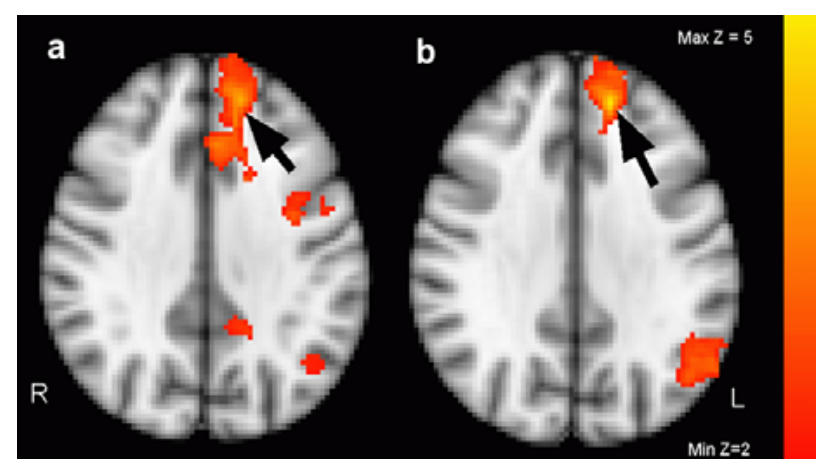

\section{FIGURE 4}

Behavior description interview activation in (a) the left hippocampal region, (b) behavioral description activation in the right hippocampal region, and (c) situational interview activation in the left parahippocampus.

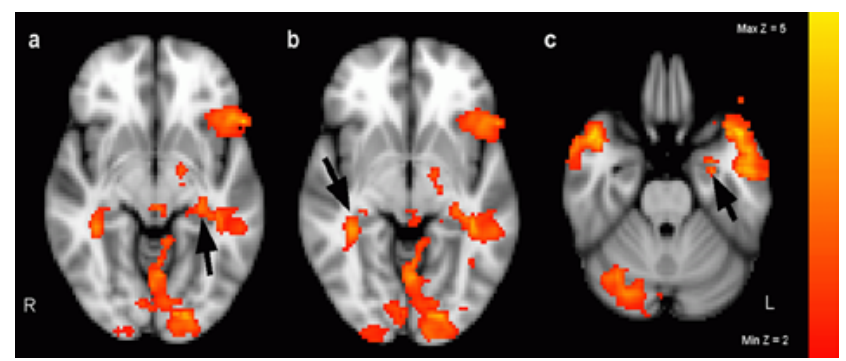

Situational interview. Interestingly, there was activation in the left amygdala in the SI that was not matched in the BDI. The amygdala is a small structure attached to the end of the hippocampus that is the emotional center of the brain (Swanson \& Petrovich, 1998). Recall that the BDI questions were presented first, implying that this was not general stress associated with the fMRI process. This activation, shown in the left side of Figure 5 (Panel a), could turn out to be a relatively interesting scientific finding.

Additionally, although the DL-PFC was activated with both blocks of questions, there was one prefrontal area that was uniquely activated in the SI. It was the left orbitofrontal region, which is shown in the right side of Figure 5 (Panel b). This activation appears to correspond with Brodmann areas 11 and 12. The term "orbitofrontal" is named as such because this region of the brain lies directly above the eye sockets (i.e., orbits). Among prefrontal regions, this one is the least understood, the most complex, and the slowest to develop and mature. Processes thought to reside there
FIGURE 5.

Situational interview activation in the (a) left orbitofrontal cortex, and (b) left amygdala.

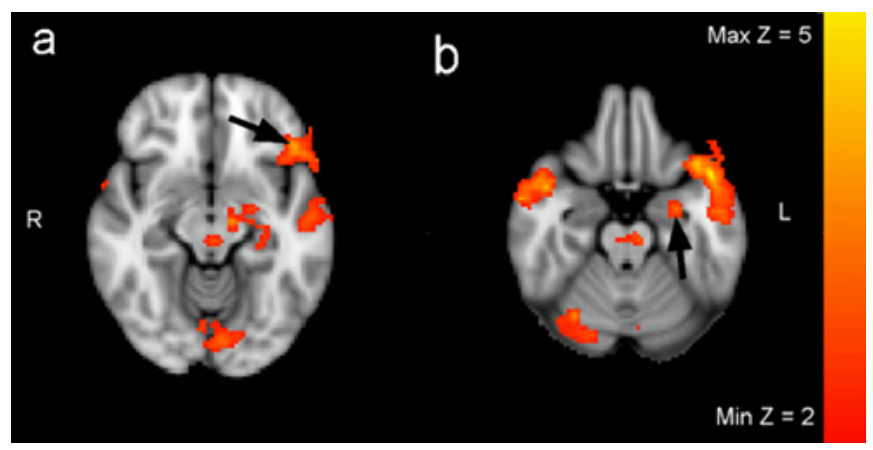

include impulse control and assessment of normative standards and "social value" (Anderson, Bechara, Damasio, Tranel, \& Damasio, 1999; see also Kringelbach, 2005), as well as adaptive learning (Schoenbaum, Takahashi, Liu, \& McDannald, 2011). In short, there is some possibility that this could be a social-processing center of the brain.

\section{DISCUSSION}

Is fMRI really a viable technique for organizational research? Ten or 20 years ago, the answer might very well have been no, particularly because scanning equipment only tended to be available in hospitals. With increased availability at larger universities, fMRI research may now be more realistic. Outlets such as the Organizational Neuroscience column in TIP have helped raise awareness of the potential of this type of research. Yet, more effort is needed, such as inclusion of neuroscience in graduate I-O content. It is not hard to envision a future where meaningful streams of neuroscience research take their place alongside those involving conventional methodologies.

I-O psychologists tend to think in terms of constructs, and a legitimate question becomes what constructs were highlighted and captured (or at least suggested) with our fMRI scans. General mental ability (GMA) is one of the most popular constructs in I-O, particularly in selection. Research suggests that it predicts both level of occupational attainment and performance within occupations and does so better than anything else including experience and personality traits (Schmidt \& Hunter, 1998; 2004). Yet, despite obvious information processing demands in both SIs and BDIs, research has failed to find much in terms of correlation with GMA (e.g., Roth \& Huffcutt, 2013).

Our findings suggest the possibility that that GMA manifests itself in distinct ways in regard to the process of responding to SI and BDI questions. Three specific regions are implicated at the present time (and more may follow): 
the dorsolateral prefrontal cortex or DL-PFC, which seems to be the most purely cognitive (e.g., working memory); the orbitofrontal cortex (OFC), which seems more attuned to processing of complex social aspects; and the hippocampus, which is clearly the dominant region for both storing and retrieving information from long-term memory. Recall the Eisenberger et al. (2005) study described much earlier where neuroticism-related activation was assessed directly, and it correlated more strongly with behavioral outcomes than a self-report measure of neuroticism. Similarly, it is possible that the level of activation in some of these regions might correlate more strongly with SI and BDI ratings than a paper-and-pencil measure of GMA. Given that activation can be expressed statistically (e.g., as a $z$ value), this type of research should not be overly difficult to do.

Another (and related) potential avenue for future research emerges from the possibility that we may have a separate region of the brain that specializes in dealing with complex social situations (the orbitofrontal cortex). This region could be the source of the tendency for interviewees to engage in the impression management tactic ingratiation with SIs (Ellis, West, Ryan \& DeShon, 2002), or at least a prime contributor thereof. Alternately, this region could relate to social intelligence, a concept that has been around for a long time (e.g., Thorndike \& Stein, 1937), yet has never been firmly established as a mainstream construct in I-O despite its obvious potential. A primary concern is whether social intelligence actually is distinct from GMA and similar mental capabilities (see Landy, 2006). It may not be, but then again, it is possible that the problem is not with the construct itself but with its measurement. fMRI provides a new and potentially promising way to measure brain activation associated with social processing directly.

Complicating the issue of construct assessment is the fact that interviews - even highly structured ones - are a method and not a direct construct measure. Contextual differences such as the type of position can influence what characteristics are embedded in interviewer ratings. The fMRI technique has the potential to highlight underlying processes that remain largely consistent across interviewees and situations. For instance, it is safe to assume that candidates process the details and parameters of each question regardless of type, and that could very well occur in the DL-PFC. Searching long-term memory for relevant experiences is the base process for BDIs and should be present regardless of the candidates, the situation, and the specific questions asked.

Limitations should be noted. First, by necessity our interview protocol deviated from traditional oral presentation and response, which makes our results more tentative. As noted earlier, however, strong activation in both Wernicke's and Brocca's areas suggested that that our participants were comprehending the questions and forming verbal responses in a very similar manner. Second, we were not able to counterbalance the presentation of SI and BDI question blocks, largely because we used hospital equipment with limited time and staff resources. We do not believe that presenting the BDI questions first had a significant impact our results, but we cannot rule that out. Utilizing university fMRI equipment in follow-up research should allow greater flexibility. Third, our sample size was small and limited to right-handed individuals. Given the strength of our findings (e.g., $z$ values in the 3-4 range), we believe them to be legitimate and generalizable. Nonetheless, our findings should be replicated with larger and more diverse samples.

Notwithstanding these limitations, we believe that our sample study not only found meaningful results that advance understanding of situational and behavior description interviews but also highlighted the potential of fMRI for investigating a wide range of organizational issues. In our way of thinking about neuroscience, becoming mainstream in industrial-organizational psychology is not a matter of "if" but rather a matter of "when." We hope that this manuscript helps pave the way.

\section{REFERENCES}

Anderson, S. W., Bechara, A., Damasio, H., Tranel, D., \& Damasio, A. R. (1999). Impairment of social and moral behavior related to early damage in human prefrontal cortex. Nature Neuroscience, 2, 1032-1037.

Baars, B. J., \& Gage, N. M. (2010). Cognition, brain, and consciousness (2nd ed.). New York, NY: Academic Press.

Brodmann, K. (1909). Localisation in the cerebral cortex. Leipzig, Germany: Johann Ambrosius Barth.

Cabeza, R., Ciaramelli, E., Olson, I. R., \& Moscovitch, M. (2008). The parietal cortex and episodic memory: An attentional account. Nature Reviews Neuroscience, 9, 613-625.

Culbertson, S. S., Weyhrauch, W. S., \& Huffcutt, A. I. (2017). A tale of two formats: Direct comparison of matching situational and behavior description interview questions. Human Resource Management Review, 27, 167-177. doi: 10.1016/ j.hrmr.2016.09.009

Dawson, M. J. (2013). Paul Lauterbur and the invention of MRI. Cambridge, MA: The MIT Press.

DeLong, M. R., Alexander, G. E., Georgopoulos, A. P., Crutcher, M. D., Mitchell, S. J., \& Richardson, R. T. (1984). Role of basal ganglia in limb movements. Human Neurobiology, 2, 235244.

Desmond, J. E., \& Glover, G. H. (2002). Estimating sample size in functional MRI (fMRI) neuroimaging studies: Statistical power analyses. Journal of Neuroscience Methods, 118, 115-128.

Diana, R. A., Yonelinas, A. P., \& Ranganath, C. (2007). Imaging recollection and familiarity in the medial temporal lobe: $A$ three-component model. Trends in Cognitive Neuroscience, 11, 379-386. 
Eisenberger, N. I., Lieberman, M. D., \& Satpute, A. B. (2005). Personality from a controlled processing perspective: An fMRI study of neuroticism, extraversion, and self-consciousness. Cognitive, Affective, \& Behavioral Neuroscience, 5, 169-181.

Ellis, A. P. J., West, B. J., Ryan, A. M., \& DeShon, R. P. (2002). The use of impression management tactics in structured interviews: A function of question type? Journal of Applied Psychology, 87, 1200-1208. doi: org/10.1037/0021-9010.87.6.1200

Epstein, R., \& Kanwisher, N. (1998). A cortical representation of the local visual environment. Nature, 392, 598-601. doi: $10.1038 / 33402$

Freeman, W. J. (2004). Origin, structure, and role of background EEG activity. Part 1: Analytic amplitude. Clinical Neurophysiology, 115, 2077-2088.

Fuster, J. M. (1997). Network memory. Trends in Neurosciences, 20, 451-459.

Gailliot, M. T. (2008). Unlocking the energy dynamics of executive functioning: Linking executive functioning to brain glycogen. Perspectives on Psychological Science, 3, 245-263. doi: 10.1111/j.1745-6924.2008.00077.x

Garey, L. J. (2006). Brodmann's localisation in the cerebral cortex. New York, NY: Springer.

Gifford, R., Ng, C. F., \& Wilkinson, M. (1985). Nonverbal cues in the employment interview: Links between applicant qualities and interviewer judgments. Journal of Applied Psychology, 70, 729-736.

Huettel, S. A., Song, A. W., \& McCarthy, G. (2009). Functional magnetic resonance imaging ( $2 \mathrm{nd}$ ed.). Sunderland, MA: Sinauer.

Huffcutt, A. I., Conway, J. M., Roth, P. L., \& Klehe, U. C. (2004). The impact of job complexity and study design on situational and behavior description interview validity. International Journal of Selection and Assessment, 12, 262-273.

Huffcutt, A. I., Culbertson, S. S., \& Goebl, A. P. (2015). The interactive influence of ambition and sociability on performance in a behavior description interview. Personnel Assessment and Decisions, 1, 30-36.

Huffcutt, A. I., Culbertson, S. S., \& Weyhrauch, W. (2013). Employment interview reliability: New meta-analytic estimates by structure and format. International Journal of Selection and Assessment, 2l, 264-276.

Huffcutt, A. I., Culbertson, S. S., \& Weyhrauch, W. S. (2014). Moving forward indirectly: Reanalyzing the validity of employment interview with indirect range restriction methodology. International Journal of Selection and Assessment, 22, 297-309.

Huffcutt, A. L, \& Roth, P. L. (1998). Racial group differences in employment interview evaluations. Journal of Applied Psychology, 83, 179-189.

Hunter, J. E., Schmidt, F. L., \& Jackson, G. B. (1982). Meta-analysis: Cumulating research findings across studies. Beverly Hills, CA: Sage.
Janz, T. (1982). Initial comparison of patterned behavior description interviews versus unstructured interviews. Journal of Applied Psychology, 67, 577-580. doi: 10.1037/00219010.67.5.577

Janz, T. (1989). The patterned behavior description interview: The best prophet of the future is the past. In R. W. Eder and G. R. Ferris (Eds.), The employment interview: Theory, research, and practice (pp. 158-168). Thousand Oaks, CA: Sage.

Kennison, S. (2013). Introduction to language development. Los Angeles, CA: Sage.

Kringelbach, M. L. (2005). The orbitofrontal cortex: Linking reward to hedonic experience. Nature Reviews Neuroscience, 6, 691-702.

Kuncel, N. R., \& Sackett, P. R. (2014). Resolving the assessment center construct validity problem (as we know it). Journal of Applied Psychology, 99, 38-47. doi: 10.1037/a0034147

Landy, F. J. (2006). The long, frustrating, and fruitless search for social intelligence: A cautionary tale. In K. R. Murphy (Ed.), A critique of emotional intelligence: What are the problems and how can they be fixed? (pp. 81-123). Mahwah, NJ: Erlbaum.

Latham, G. P., Saari, L. M., Pursell, E. D., \& Campion, M. A. (1980). The situational interview. Journal of Applied Psychology, 65, 422-427. doi 10.1037/0021-9010.65.4.422

Machielsen, W. C., Rombouts, S. A., Barkhof, F., Scheltens, P., \& Witter, M. P. (2000). FMRI of visual encoding: Reproducibility of activation. Human Brain Mapping, 9, 156-164.

Matthews, G., \& Gilliland, K. (1999). The personality theories of H. J. Eysenck and J. A. Gray: A comparative review. Personality \& Individual Differences, 26, 583-626.

Mégevand, P., Groppe, D. M., Goldfinger, M. S., Hwang, S. T., Kingsley, P. B., Davidesco, I., \& Mehta, A. D. (2014). Seeing scenes: Topographic visual hallucinations evoked by direct electrical stimulation of the parahippocampal place area. Journal of Neuroscience, 34, 5399-5405. doi: 10.1523/jneurosci.5202-13.2014

Miller B. L., \& Cummings, J. L. (2007, Eds.). The human frontal lobes: Functions and disorders. New York, NY: Guilford.

Miyake, A., Friedman, N. P., Emerson, M. J., Witzki, A. H., \& Howerter, A. (2000). The unity and diversity of executive functions and their contributions to complex "frontal lobe" tasks: A latent variable analysis. Cognitive Psychology, 41, 491000.

Nisiyama, M., \& Ribeiro-do-Valle, L. E. (2014). Relative performance of the two hands in simple and choice reaction time tasks. Brazilian Journal of Medical and Biological Research, 47, 80-89.

Poldrack, R. A., Mumford, J. A., \& Nichols, T. E. (2011). Handbook of functional MRI data analysis. Cambridge, UK: Cambridge University Press.

Rinck, P. A. (2001). Magnetic resonance in medicine (4th ed.). Minusio, Switzerland: EMRF Foundation. 
Roth, P. L., \& Huffcutt, A. I. (2013). A meta-analysis of interviews and cognitive ability: Back to the future? Journal of Personnel Psychology, 12, 157-159.

Russell-Chapin, L., Kemmerly, T., Liu, W.C., Zagardo, M., \& Chapin, T. (2013). The effects of neurofeedback in the default mode network: Pilot study results of mediated children with ADHD. Journal of Neurotherapy, 17, 35-42.

Schmidt, F. L., \& Hunter, J. E. (1998). The validity and utility of selection methods in personnel psychology: Practical and theoretical implications of 85 years of research findings. Psychological Bulletin, 124, 262-274.

Schmidt, F. L., \& Hunter, J. (2004). General mental ability in the world of work: Occupational attainment and job performance. Journal of Personality and Social Psychology, 86, 162-173. doi: 10.1037/0022-3514.86.1.162

Schoenbaum, G., Takahashi, Y., Liu, T., \& McDannald, M. (2011). Does the orbitofrontal cortex signal value? Annals of the New York Academy of Sciences, 1239, 87-99. doi:10.1111/ j.1749-6632.2011.06210.x

Swanson, L. W., \& Petrovich, G. D. (1998). What is the amygdala? Trends in Neurosciences, 21, 323-331. doi:10.1016/S01662236(98)01265-X

Talairach, J., \& Tournoux, P. (1988). Co-planar stereotaxic atlas of the human brain. New York, NY: Thieme Medical Publishers.

Taylor, P., \& Small, B. (2002). Asking applicants what they would do versus what they did do: A meta-analytic comparison of situational and past behaviour employment interview questions. Journal of Occupational and Organizational Psychology, 75, 277-294.

Thorndike, R. L., \& Stein, S. (1937). An evaluation of attempts to measure social intelligence. Psychological Bulletin, 34, 275285.

Thulborn, K. R., Waterton, J. C., Matthews, P. M., \& Radda, G. K. (1982). Oxygenation dependence of the transverse relaxation time of water protons in whole blood at high field. Biochimica et Biophysica Acta (BBA), 714: 265-270. doi:10.1016/0304-4165(82)90333-6

Trafton, A. (2012, Jan. 9). How does our brain know what is a face and what's not? MIT News. Retrieved from https://medicalxpress.com/news/2012-01-brain.html

Ward, M. K. (2017, Fall). Organizational neuroscience: An overview of ways entrepreneurship is capitalizing on neuroscience. The Industrial-Organizational Psychologist, 55(2). Retrieved from http://my.siop.org/tip/jan18/editor/ ArtMID/13745/ArticleID/121/Organizational-Neuroscience-An-Overview-of-Ways-Entrepreneurship-Is-Capitalizing-on-Neuroscience-Inspiration-for-I-O-Psychology

Ward, M. K., \& Becker, B. (2013, July). Organizational neuroscience. The Industrial-Organizational Psychologist, 51(1): 7780.
Ward, M. K., \& Becker, B. (2014, October). Organizational neuroscience: Neuroscience and leadership development. The Industrial-Organizational Psychologist, 52(2), 55-59.

Ward, M. K., \& Becker, B. (2015, April). Organizational neuroscience: Neuroscience and leadership development. The Industrial-Organizational Psychologist, 52(4), 16-21.

Weiner, K. S., \& Grill-Spector, K. (2010). Sparsely-distributed organization of face and limb activations in human ventral temporal cortex. Neuroimage, 52, 1559-1573.

Zhu, X., Ward, M. K., \& Becker, B. (2016, Summer). Where have we been with organizational neuroscience? A review of past themes and visions of the future. The Industrial-Organizational Psychologist, 54(1). Retrieved from http://www.siop. org/tip/july16/on.aspx 